\title{
The Most Effective Treatment or Intervention for Treating Chinese Elderly Depression: a Literature Review
}

\author{
Baljit Kaur ${ }^{1,2}$ Vivian Lou, W. Q. ${ }^{3}$ \\ ${ }^{1}$ Lecturer, Nursing, \\ The Open University of Hong Kong \\ Hong Kong \\ bkaur@ouhk.edu.hk \\ ${ }^{2}$ The University of Hong Kong \\ baljit@connect.hku.hk \\ ${ }^{3}$ Department of Social Work and Social Administration \\ The University of Hong Kong \\ wlou@hkucc.hku.hk
}

\begin{abstract}
Background: There is an increase in the percentage of Chinese elderly population with depression globally which may be contributed by financial stain, poor living conditions, and poor social support with friends and relatives, absence of carer and precipitating poor health condition and different therapy had been used for them.

Objectives: This literature review was conducted to evaluate different treatment and interventions that had been used on Chinese elderly population with depression.

Methodology: A systematic searching process from January, 2013 to June, 2013. Key word used is elder, geriatric, senile, old age, aged 60 or above, depress, depression, depressive symptoms and Chinese.

Results: 84 studies were retrieved and it shows that Tai Chi is most effective in treating depression symptoms, Qigong is most effective in treating the associated factors of depressive symptoms and lastly Qigong is also the most effective therapy in treating the general wellbeing of the outcomes of depression.
\end{abstract}

Keywords: Elder, Geriatric, Senile, Older, Old age, Aged 60 or above, Depress, Depression, Depressive symptoms, Chinese.

\section{Introduction}

Aging is a lifelong process and it begins before when we were born and continues throughout life. It can be regarded as a life-course perspective and that elderly are not one homogenous group and the diversity in everyone changes with the increase in age. As the total percentage of elderly population is increasing ${ }^{[1,2]}$ thus awareness is also marked on their process of aging in order to provide, maintain and gain a fruitful older periods of life.

In the past, aging and health problem are placed together due to the advancement in technology, people are accepted to live longer and due to the increase in the number of elderly, concerns and awareness are highlighted on them. Nowadays, aging is referred to as an issue that is one of the most emphatically as a challenge and opportunity ${ }^{[3]}$. This group of elderly was once major contributing factor and manpower in the development of the present society and nation thus they must not be neglected. Therefore, services, therapy and supports for elderly should be able to provide a supportive environment and offering healthy choices at all different stages of aging.

In the means of providing a healthy and fruitful older life period to this group of elderly and the concept of "active aging" was introduced by the World Health Organization (WHO) as guidance to different sectors in planning their policy and services to the elderly community ${ }^{[4]}$. The previous concept of referring whether elderly is healthy or not, does only focuses on the physical health of the individual which stands of being free from illness and physical disability ${ }^{[4]}$. In the year of 2002, WHO had introduced a new definition of health which is defined as a state of complete physical, mental and social well-being and merely the absence of disease or infirmity and the concept of "active aging" was also widely publicized $^{[4]}$.

The concept of "active aging" is used commonly and readily in promoting aging in elderly. WHO adopted the term "active aging" in the late 1990s and defines "active aging" concept as "the process of optimizing opportunities for health, participation, and security to enhance quality of life as people age ${ }^{[4]}$. The concepts also helps individual to realize their potential physical, social and mental well-being throughout life and participate accordingly to their needs, desires and capacities ${ }^{[5]}$.

The definition of "active" in "active aging" can be defined as the participation of social, economic, cultural, spiritual and civic affairs by individual ${ }^{[5]}$. The determinants of "active aging" determined by WHO (2002) are health and social services, behavioral determinants, personal determinants, physical environment, social determinants and economic determinants ${ }^{[4]}$. Elderly can remain active even though they 
retire from work, they can still perform an active role in their families, peers, communities and nations and the goal of "active aging" is to extend their respective healthy life and quality of life.

The concept of "active aging" approach can also be used in many different settings of elderly life. They had their own independence, participation, dignity care and self-fulfillment ${ }^{[4]}$. Active aging can also be used in better management of the psychological health of elderly. Individually, elderly should be independent, have active participation, dignity and selffulfillment should be provided and experienced by elderly when they are receiving care and treatment for their psychological health. This is also one of the major reasons why we need to determine what is being effective in treating the various health conditions that elderly experienced both physically and mentally.

\section{Chinese Older Adult Depressive Symptoms}

Depressive symptoms are a common illness seen in elderly population. It had been estimated that there is 340 million of people in the world who are suffering from depressive symptoms ${ }^{[6]}$. There are a significant number of Chinese elderly who are suffering from depressive symptoms as well and depression is also one of the most common mental health problems among Chinese elderly in different part of the world. Different researches had proofed that the prevalence of having depressive symptoms increases with age ${ }^{[7]}$ though the rate of prevalence may be due to the characteristics of the sampling of the Chinese elderly. Among Chinese elderly who have chronic illness or are patients, it had been found that in Shanghai, China $50 \%$ of the elderly process depressive symptoms ${ }^{[8]}$; in Hong Kong, it ranges from 8 to $35 \%$ of Chinese elderly patients have depressive symptoms ${ }^{[9,10]}$. Among the community dwelling Chinese elderly who presents with depressive symptoms are $13 \%$ in the Liverpool area of the United Kingdom ${ }^{[12]}, 16 \%$ in Manhattan, New York and Hong Kong is having $29 \%$ of elderly female and $41 \%$ of elderly male is suffering depressive symptoms ${ }^{[11]}$ and lastly in another research with sample recruited in suburban region of Tai Wan, it shows that $35 \%$ of the Chinese elderly process with depressive symptoms. Though, the characteristics of the Chinese elderly sample may affect the results of the high prevalence rate of processing depressive symptoms among Chinese elderly but it can also be alerted that there is a need to treat this large group of Chinese elderly processing depressive symptoms. It had been alerted that there is a need to understand the reason of such a high prevalence rate of Chinese elderly depressive symptoms and the contributing factor that may hinder the appropriate services and therapy being able to be provided to them and Chinese culture value may contribute to one of these factors.

\section{Chinese Culture versus Depressive Symptoms}

Cultural factor plays one of the hinder factors of prohibiting essential care need to be provided taken among Chinese elderly who are suffering from depressive symptoms and it is also an important social determinant of health ${ }^{[13]}$ and cultural influences behaviors, health beliefs, illness prevalence, illness expressions and outcomes ${ }^{[14]}$.

Different culture and ethic group have different expressions for emotional experiences and self-rating scales which may have biased by different cultural concepts and idioms ${ }^{[15,16]}$.

\section{Conceptualization}

In tradition Chinese people thinks that health is the harmony between two opposite forces of "yin" and "yang" within the body against the body and its outside environment. Among Chinese culture, depression is also called "yiyu" and according to Chinese dialect, "yiyu" is interpreted as depression in western culture. Yu" means depression in traditional Chinese medicine which implies the individual is experiencing a stage of stagnation [17]. "Stagnation" or "yu" means that the individual's yu is not flowing, entangled, blocked or clogged which stagnation the functional activity of "qi" ${ }^{[18]}$. When there is stagnation of "qi", the individual will be experiencing mind/body dysfunctions such as disrupted emotions with experiencing anger most of the time, sleeping disorders, frequent complain of dizziness, fatigue and decrease in appetite. Thus, "yiyu" carries a definition of oneself experiencing depression. "Yiyu" or "stagnation of qi" can be healed or cure by treating the individual with herbal medicine and acupuncture ${ }^{[19]}$. But it is believed that the real method of cure for "yiyu" is that there should be de-stagnation of the negative emotions of the individual ${ }^{[20,21]}$.

Chinese elderly population are still rooted by the traditional Chinese philosophy, the teaching of Confucianism and Taoism which promote moderation, calmness and forbearance and thus can influence pattern of reveal of their depressive symptoms and effectiveness ${ }^{[22]}$. A study done in Hong Kong shows that Chinese elderly patients with depressive symptoms are seen in general practice in Hong Kong was more likely to complain that they have psychological and depressive symptoms when they are interviewed by the researcher than to the physician during consultation ${ }^{[22]}$.

\section{Manifestation-Somatization}

Somatization is often another way of Chinese elderly to express their depressive symptoms ${ }^{[23]}$. Chinese ethic group often reports more somatic symptoms than other ethnic groups ${ }^{[24]}$. When discovering the reason behind this, it had been found that in traditional Chinese medicine, physical symptoms are often

more salient than other psychological factors and consequently that Chinese elderly often express their mental/psychological problems as physical symptoms ${ }^{[24]}$.

\section{Stigma and Discrimation of Older Adult with Depressive Symptoms}

The awareness of the importance of mental health had increased but the stigma and discrimination among mental illness sufferer and non-sufferer still present in most nations and community and consequently this had hindered in detecting and treating mental illness. 
Stigma and discrimination are often associated with mental disorders which cause suffering, disability and economic losses. Stigma is a course of action that the individual is rendered shameful and discriminate by others in the community and discrimination is the distinction and preference that the individual has taken from enjoying the equal rights as others can. Every individual who suffers from mental health problem should be treated with humanely and obtain respect and adhere dignity from all other people. Defining and imposing a stigma or discrimination on any person is not right to do so.

Stigma of mental health also arises in elderly population as there is often ignorance and misconceptions of old age and their mental health disorders and their therapy. Aging is often referred to someone who is weak, ill and unproductive. People often carry the taboo that an individual with mental disorders are dangerous and have self-harm beliefs. Stigma and discrimination toward elderly with mental disorders do not only exist in normal citizens in the community but it also exists in professionals as well. Professionals often presents with negative attitude toward elderly with mental disorders and to those who provide services to them. The public media also plays an important role in enhancing the negative attitude toward elderly with mental disorders.

Due to the fact of the present of stigma and discrimination of the process mental illness and thus this had lead to the problem that Chinese elderly depressive symptoms is hard to tackle because Chinese individuals are often more likely to suppress the expression of emotions and use more somatic symptoms to convey emotional distress ${ }^{[25]}$. In addition, being a psychiatric patient carries stigma for Chinese elderly and may adversely affect their own marriage or career opportunities and even their family members' careers or marriages. In Chinese culture, physical illness is viewed as beyond one's control and therefore it is more socially acceptable ${ }^{[23,25]}$ and Chinese elderly are often reluctant to seek help even if they have depressive symptoms and avoid receiving appropriate treatment. Therefore, the most prompt and suitable therapy should be provided to this vulnerable group of elderly before they change their mind of receiving the therapy.

\section{METHODOLGY}

A systematic searching process was carried out to search all the published studies that were related to the study question from January, 2013 to June, 2013. The criteria for inclusion of the studies in this study had also been used by other research studies on elderly depression therapy and intervention [26,27, 28, 29] are as follows. 1) The study will considered all potential articles that were published before September, 2009; 2) They must be published and written in English ${ }^{[26]} 3$ ) studies were published in peer-reviewed journals; 4) a prospective test of any kind for Chinese elderly depression; 5) report a mean subject age of 60 years or older or a lower limit on age no less than 60 years ${ }^{[29]}$; 6) the results from the studies should report at least one of the following outcomes as caregiver's burden, depression, measures of psychological well-being such as life satisfaction, morale, self-esteem, happiness ${ }^{[29]}$. Key words that were adopted in the searching are elder, geriatric, senile, older old age, aged 60 or above, depress, depression, depressive symptoms and Chinese. The effectiveness of different therapy and intervention were compared against each other regarding their effectiveness on different of aspects of depression such as depression symptoms, associated factors of depression and general well-being of the outcomes of depression. The same therapy and intervention of depression were also compared with the effectiveness on western older population.

\section{RESULT}

Under the study question of the different treatment and intervention for Chinese elderly with depression, 84 related studies were retrieved which met the inclusion and exclusion criteria mentioned above. 38 of studies were discussing only the causative factors of Chinese elderly and no therapy was given to them therefore it was excluded from this metaanalysis paper. 10 of the studies were discussing the causes of actions that the Chinese elderly suffering from depressive symptoms experience and no therapy was given to them. 15 of the included studies was discussing on the depressive symptoms assessment tools only and lastly 11 of them were published in Chinese dialect with only supported by English abstract. Therefore, there were only 10 studies that had met the inclusion and exclusion criteria and were included in doing this critical review paper.

The ten studies demonstrated different treatment and intervention that had been used in treating Chinese elderly depression which are Cognitive Behavioral Therapy (CBT), reminiscence therapy, peer counseling therapy, Qigong, Tai Chi and light therapy.

\begin{tabular}{|l|l|}
\hline $\begin{array}{l}\text { Scholar name and } \\
\text { publication }\end{array}$ & Intervention/ therapy \\
\hline Dai et al., 1999 & CBT \\
\hline Tsang et al., 2003 & Qigong \\
\hline Tsai et al., 2004 & Light \\
\hline Chou et al., 2004 & Tai Chi \\
\hline Wang, 2005 & Reminiscence \\
\hline Wang et al., 2005 & Reminiscence \\
\hline Tsang et al., 2006 & Qigong \\
\hline Ho, 2007 & Peer Counseling \\
\hline Chiang et al., 2009 & Reminiscence \\
\hline $\begin{array}{l}\text { Gallagher-Thompson } \\
\text { et al., 2007 }\end{array}$ & CBT \\
\hline
\end{tabular}

\section{Psychotherapy}

The publication related to the study question is limited but with all the published studies that was retrieved, it had been noted that psychology therapy $[27,30,31,32]$ is often most commonly selected as the means of treatment and intervention and this may be due to the long established of the therapy that 
had been used on depressive symptoms and being widely established in Western elderly population as well.

\section{Cognitive Behavioral Therapy $(\mathrm{CBT})$}

Cognitive Behavioral Therapy (CBT) was used in two of the recruited studies ${ }^{[30,32]}$ and it was critique against two studies in western elderly population ${ }^{[30]}$. It shows that CBT is relatively more effective and applicable to be used on western elderly population with depression.

\section{Reminiscence Therapy}

Three of the ten recruited studies ${ }^{[31]}$ were using reminiscence therapy to treat depression and which had showed a positive results of the therapy. The application of the therapy was critique with another study done on western elderly with depression and it shows that reminiscence therapy was more effective and applicable to be used on Chinese elderly than the western elderly population.

\section{Tai Chi}

Among different depression therapy and intervention in the Chinese elderly, it shows that Tai Chi ${ }^{[33]}$ is most effective in treating Chinese elderly depression and it was also compared against western population ${ }^{[28]}$, which shows that the therapy is more effective to be used on Chinese elderly.

\section{Qigong}

Qigong ${ }^{[34]}$ is most effective in treating the associated factors of depression among Chinese elderly with depressive symptoms and lastly Qigong is also the most effective therapy and intervention in treating the general well-being of the outcomes depression among Chinese elderly with depressive symptoms.

\section{IMPLICATIONS}

Focusing on only the effectiveness of different theory to treat Chinese elderly depression will not be able to benefit individual in long term with a combination of policy planning, health promotion programme and publicizing on family values.

\section{Policy Planning}

Though, we had a picture of different therapies that had been used so far on treating Chinese elderly depressive symptoms but it is also important to remember focusing on only providing treatment is not enough and the results may not everlasting as well and the development of policy should also be amended and improve as well. Policy should not only focus on the mental health disorders instead they should investigate and act against the reasons of poor mental health in the community and society.

\section{Health Promotion Programme}

The principles and knowledge of public health and health promotion can be used in the application of mental health in the society just as those health promotion programmes of cardiovascular disease. Health promotion programme involves creating a supportive environment in the society to support promotion of healthy mental health activities and healthy lifestyles. The concept of mental health promotion aims to provide a positive view and attitude on mental health of every individual. A study conducted in Hong Kong stated that social support from family is important for Chinese elderly in Hong Kong.

\section{Family Value}

Asian family values appreciate the value of family care living together does not imply that the elderly is receiving the appropriate care and sufficient support for them. There is little is known about the needs of the elderly such as his and her preferences, their choice of living arrangement and their mutual benefits. Quality of life of elderly is also another aspect closely related to the health of the elderly. Health at elderly is link with lifelong health.

\section{LIMITATIONS OF THE STUDY}

The limitations of the study can be divided into two major categories as publication bias and author limitations.

\section{Publication Bias}

In this study, different publication were also raised such as inclusion criteria bias, selector bias, extractor bias as this thesis is an individual work and personal preferences on selecting studies is often raised. Other bias that was come across in this study are quality bias, reporting bias, recording bias, geographic bias and follow-up time bias as the methodology, uses data and information publish in other studies and the author of this thesis had not ask the author of the included studies to verify the result published in these studies.

\section{Author Limitation}

The author of this thesis do not know how to read and write Chinese dialect and this had hinder the author to retrieve relevant studies that discuss the therapy for Chinese elderly with depressive symptoms. There are numerous studies that are published in China that studies depressive symptoms therapy and usage of Chinese medication was widely used as well. Nevertheless, all these studies are published in Chinese dialect and were not able to be used and included in this thesis.

\section{ACKNOWLEDGMENT}

I would like to Acknowledge Dr. Vivian Lou for giving me all the guidance in doing this systematic review.

\section{REFERENCES}

[1] Bernard, M. \& Philips, J. (2000). The challenge of aging in tomorrow's Britain. Aging and Society, 20, 33-54.

[2] Kinsella, K. \& Phillips, D. R. (2005) Global aging: The challenge of success. Population Bulletin, 60 (1), 3-5. 
[3] United Nations (2002) Johannesburg Plan of Implementation. New York: United Nations.

[4] World Health Organization (2002) Active Ageing: A Policy Framework. Geneva: World Health Organization.

[5] Robinson, M. et al., (2007) Global Health \& Global Aging. United States of America: Jossy-Bass.

[6] Murray, C. J. L. \& Lopez, A. D. (1996) The Global Burden of Disease: A Comprehensive Assessment of Mortality and Disability from Diseases, Injuries, and Risk Factors in 1990 and Projected to 2020, vol1. World Health Organization. Cambridge, Mass: Harvard University Press.

[7] Kua, F.H. (1989) Depressive disorder in elderly Chinese people. Acta Psychiatry Scandal, 81, 386-88.

[8] Pan, M. (1991) Mental Health Problems of the Elderly Staying with the family. Shanghai: Shanghai Gerontological Association.

[9] Lam, A. \& Chan, W. M. (2003) Depression in Elderly. Public Health and Epidemiology Bulletin. Department of Health Hong Kong, China, 12(5), 53-58.

[10] Lee, C.K. (2003) Prevalence of and factors associated with depression among community elderly people using mobile health clinic. The University of Hong Kong. M Med Sc Dissertation.

[11] Woo, J., Ho, S.C., Lau, J. et al., (1994) The prevalence of depressive symptoms and predisposing factors in an elderly Chinese population. Acta Psychiatric Scandal, 89, 8-13.

[12] McCracken, C. F. M. et al., (1997) Prevalence of dementia and depression among elderly people in Black and ethnic minorities. British Journal Psychiatry, 171, 269-73.

[13] Lai, D. W. L. (2004) Impact of culture on depressive symptoms of elderly Chinese immigrants. Canada Journal of Psychiatry, 49 (12), 820827.

[14] Kaslow, N. J. et al., (2007) Health care for the whole person: research update. Professional Psychology: research and Practice, 38(3), 278-289.

[15] Tanaka-Matsumi, J. \& Marsella, A. J. (1976) Cross-cultural variations in the phenomenological experience of depression: Word association. Journal of Cross cultural Psychology, 7, 379-396.

[16] Zheng, Y. et al. (1986) Styles of verbal expression of emotional physical experiences: a study of depressed patients and normal controls in China. Cultural Medicine of Psychiatry, 10, 231-243

[17] Ho, Y. W. (1995) Traditional Chinese Psychiatry, Shanghai, Shanghai Popular Science Publishing (In Chinese).

[18] Yuen, Y. C. et al., (1997) Chinese-English Dictionary of Traditional Chinese Medicine, Beijing, People's Health Publishing.

[19] Cheung, B. Y. (1997) Chinese Medicine Internal Medicine, Shanghai, Shanghai Science and Technology Publishing (in Chinese).

[20] Mao, Y. et al., (2004) 'A stagnation model for depression: Apply TCM concept in treating depression'. International Journal of Psychology, 39(5-6 (Suppl.)), 597.

[21] Ng, S. M. (2003) Chinese Medicine Yangsheng and Body/Mind/Spirit Wellness. Veritas Book House: Hong Kong (in Chinese).

[22] Cheung, F. M. et al., (1981) Somatization among Chinese depressions in general practice. International Journal Psychiatry Medicine, 1980-1981, $10,361-374$.
[23] Mak, W. W. \& Zane, N.W. (2004) The phenomenon of somatization among community Chinese Americans. Social Psychiatry \& Psychiatric Epidemiology, 39, 967-974.

[24] Chan, B. \& parker, G. (2004) Some recommendations to assess depression in Chinese people in Australasia. Australia New Zealand Journal of Psychiatry, 38 (3), 141-147.

[25] Suen, L. J. \& Tusaie, K. (2004) Is somatization a significant depressive symptom in older Taiwanese Americans? Geriatric Nursing, 25, 157163.

[26] Cole, M. G. \& Dendukuri, N. (2003) Risk factors for depression among elderly community subjects: a systematic review and meta-analysis. American Journal of Psychiatry, 160 (6), 1147-1156.

[27] Cook, A. J. (1998) Cognitive-Behavioral pain management for elderly nursing homw residents. Journal of Gerontology, 538, 51-59.

[28] Fuzhong, L. et al., (2001) Enhancing the psychological well-being of elderly individuals through Tai Chi Exercise: a latent growth curve analysis. Structural Equation Modeling, 8 (1), 53-83.

[29] Thompson, L. W. (2001) Comparison of desipramine and cognitive/behavioral therapy in the treatment of elderly outpatients with mild-to-moderate depression. American Journal of Geriatric Psychiatry, 9 (3), 225-240.

[30] Dai, Y. et al., (1999) Cognitive Behavioral Therapy of Minor Depressive Symptoms in Elderly Chinese Americans: A Pilot Study. Community Mental Health journal, 35 (6), 537-542.

[31] Chiang, K. J. et al., (2009) The effects of reminiscence therapy on psychological well-being, depression and loneliness among the institutionalized aged. International Journal of geriatric Psychiatry.

[32] Gallagher-Thompson, D. et al., (2007) Impact of In-Home Behavioral Management Versus Telephone Support to Reduce Depressive Symptoms and Perceived Stress in Chinese Caregivers: results of a Pilot Study. The American Journal of Geriatric Psychiatry, 15 (5), 425-434.

[33] Chou, K. L. et al., (2004) Sources of income and depression in elderly Hong Kong Chinese: mediating and moderating effects of social support and financial strain. Aging \& Mental Health, 8(3), 212-221.

[34] Tsang et al., (2006) Effect of a qigong exercise programme on elderly with depression. International Journal of Geriatric Psychiatry, 21, 890897.

Baljit Kaur had obtained her Bachelor of Science (Nursing) (Hons) degree from Oxford Brooks University. She had obtained two master degrees. One of the master degree is in Master of Science (Nursing) from Oxford Brooks University and the other master degree is from The University of Hong Kong in Master of Social Science (Gerontology). She is now currently a $\mathrm{PhD}$ candidate in The University of Hong Kong. Her major interest in research is transcultural nursing, nursing education, ethical and legal nursing, simulation education, primary health care and gerontology.

Vivian Lou is an assistant professor of Faculty of Social Work and Social Adminstration in The University of Hong Kong. She is specialized in social gerontology including psychological and spiritual wellbegin, social support, long-term care. Family care giving and grandparenting. 